\title{
Increased hippocampal-inferior temporal cortex white matter connectivity following donepezil treatment in patients with mild cognitive impairment: A diffusion tensor probabilistic tractography study
}

Gwang-Won Kim

Chonnam National University

Kwangsung Park

Chonnam National University Hospital, Chonnam National University Medical School

Gwang-Woo Jeong ( $\sim$ gwjeong100@hanmail.net)

Chonnam National University Hospital, Chonnam National University Medical School

\section{Research Article}

Keywords: diffusion tensor imaging scalars, donepezil treatment, hippocampus-related networks, mild cognitive impairment, probabilistic tractography

Posted Date: October 13th, 2021

DOl: https://doi.org/10.21203/rs.3.rs-954650/v1

License: (9) This work is licensed under a Creative Commons Attribution 4.0 International License. Read Full License 


\section{Abstract}

The incidence of Alzheimer's disease (AD) has been increasing each year; however, few methods are available to identify the effects of treatment for AD. Defective hippocampus has been associated with mild cognitive impairment $(\mathrm{MCl})$, an early stage of $A D$. However, the effect of donepezil treatment on hippocampus-related networks is unknown. The purpose of this study was to evaluate the hippocampal white matter (WM) connectivity following donepezil treatment in patients with $\mathrm{MCl}$ using probabilistic tractography, and to further determine the WM integrity and changes in brain volume. Magnetic resonance imaging and diffusion tensor imaging (DTI) data of patients with $\mathrm{MCl}$ before and after 6month donepezil treatment were acquired. Volumes and DTI scalars of 11 regions of interest comprising the frontal and temporal cortices and subcortical regions were measured. Seed-based structural connectivity analyses were focused on the hippocampus. Compared with healthy controls, patients with $\mathrm{MCl}$ showed significantly decreased hippocampal volume and WM connectivity with the superior frontal gyrus, as well as increased mean diffusivity (MD) and radial diffusivity (RD) in the amygdala $(p<0.05$, Bonferroni-corrected). After six months of donepezil treatment, patients with $\mathrm{MCl}$ showed increased hippocampal-inferior temporal gyrus (ITG) WM connectivity $(p<0.05$, Bonferroni-corrected), which was normalized to the healthy control. These findings will be useful in developing theories to describe the etiology of $\mathrm{MCl}$ and the therapeutic role of anticholinesterases.

\section{Introduction}

Alzheimer's disease (AD) is characterized by progressive deterioration in learning and memory ability, which typically progresses slowly in three general stages: preclinical $A D$, mild cognitive impairment (MCl), and $\mathrm{AD}$-dementia ${ }^{1}$. $\mathrm{MCl}$ can be defined as cognitive decline greater than expected for individual age and education, without interfering with activities of daily living 2,3 . Approximately $10-15 \%$ of patients with $\mathrm{MCl}$ progress to $A D$ each year, whereas only $1-2 \%$ of individuals with normal cognitive level develop $A D^{4,5}$. Early detection of $\mathrm{MCl}$ and intervention are essential to predict and prevent $\mathrm{AD}$.

Recent advances in neuroimaging reported the effect of structural and functional abnormalities in the brain on $\mathrm{MCl}$, suggesting abnormalities in the medial temporal lobe including hippocampus in patients diagnosed with $\mathrm{AD}$. Hippocampal atrophy has been specifically implicated in $\mathrm{MCl}$ and $\mathrm{AD}$. A structural magnetic resonance imaging (MRI) study ${ }^{6}$ revealed decreased gray matter (GM) volume in the hippocampus, specifically in the right subiculum and left cornu ammonis (CA3). A similar study ${ }^{7}$ suggested that decreased volumes involving the hippocampus and hippocampal-precuneus/posterior cingulate cortical tracts was associated with early signs of $A D$ in patients diagnosed with $\mathrm{MCl}$. Patients with $\mathrm{MCl}$ showed significantly decreased direct functional connectivity from the left hippocampus to the right inferior temporal gyrus, right middle temporal gyrus, right parahippocampal gyrus, and part of the medial frontal cortex compared with normal controls ${ }^{5}$.

It is important to screen and treat $\mathrm{MCl}$ at an early stage before the development of $A D$. Treatment with acetylcholinesterase inhibitors (AChEls) in patients with $\mathrm{MCl}$ prevents the breakdown of acetylcholine 
(ACh) and increases cholinergic transmission, resulting in improved cognitive function ${ }^{6,8}$. Donepezil is the most frequently prescribed drug clinically to inhibit acetylcholinesterase activity in the cerebral cortex and hippocampus of the rat brain, revealing increased ACh activity in the brain areas associated with

cognitive function $^{9-11}$. A functional magnetic resonance imaging (fMRI) study ${ }^{12}$ reported increased medial temporal lobe activation and improved task-related connectivity of cholinergic networks after approximately 3 months of cholinergic enhancement with donepezil in patients with $\mathrm{MCl}$. A similar study ${ }^{13}$ revealed increased activity in the ventrolateral prefrontal cortex during visual memory task after 6-month donepezil treatment of $\mathrm{MCl}$.

Recent studies ${ }^{14}$ have shown that complex networks along with diffusion-weighted imaging (DWI) are effective and promising for early detection of changes in structural pathology of patients with AD. White matter (WM) degeneration occurs early in $A D$ and is useful in evaluating pathologic progression before the disease is clinically evident ${ }^{15,16}$. Probabilistic tractography in diffusion tensor imaging (DTI) has recently been used increasingly for the detection of WM integrity of an entire bundle, facilitating evaluation of structural connectivity by estimating the likelihood of connection between two areas of the brain ${ }^{16,17}$. The most prominent structural changes in $A D$ occur initially in hippocampus. A positron emission tomography (PET) study ${ }^{18}$ reported that reduced hippocampal connectivity occurs predominantly in the AD connectome, correlating with hippocampal tau in $\mathrm{MCl}$. A structural study investigating the interaction between hippocampus and cortical/subcortical regions using probabilistic tractography following donepezil treatment has yet to be reported. Identifying objective predictors of WM connectivity in $\mathrm{MCl}$ can contribute to data-driven approaches aimed at $A D$ prevention.

The purpose of this study was to evaluate the hippocampal white matter connectivity following donepezil treatment in patients diagnosed with $\mathrm{MCl}$ using probabilistic tractography, and to further assess the WM integrity and changes in brain volume.

\section{Materials And Methods}

\section{Participants}

Patients with $\mathrm{MCl}$ were inpatients or outpatients of the $\mathrm{CNUH}$. Ten patients diagnosed with $\mathrm{MCl}$ (mean age $=72.4 \pm 7.9$ years) underwent MR examination before (baseline) and after (follow-up) 6 months of donepezil treatment. The control group included nine sex- and age-matched healthy controls (mean age $=$ $70.7 \pm 3.5$ years), who were recruited via advertisements.

Patients with $\mathrm{MCl}$ were recruited based on the following criteria ${ }^{6,16,19}$ : (1) Alzheimer-type $\mathrm{MCl}$ according to both the DSM-IV and the National Institute of Neurological and Communicative Diseases and StrokeAlzheimer Disease and Related Disorders Association (NINCDS-ADRDA) criteria; (2) no history of $\mathrm{MCl}$ treatment and other neurological or psychiatric illnesses; (3) a score of 0.5 or 1 on the Clinical Dementia Rating (CDR) scale; (4) a score less than 26 on the Korean version of the Mini-Mental State Examination (K-MMSE); (5) reconfirmation of the typical symptom severity including changes in cognition recognized 
by the affected individual or observers, objective impairment in one or more cognitive domains, functional independence, and absence of dementia. After performing the first MR examination, the patients received $5 \mathrm{mg} /$ day of Aricept $^{\circledR}$ (donepezil hydrochloride; Pfizer Inc., New York, NY) for the first 28 days and 10 $\mathrm{mg} /$ day thereafter. The treatment duration for the patients was $194.0 \pm 29.5$ days, without any side effects, such as agitation, gastrointestinal bleeding, and stomach ulcer. Healthy controls were selected based on the following criteria: (1) no AD based on both the DSM-IV and the NINCDS-ADRDA criteria; (2) a score greater than 26 on the K-MMSE; and (3) no history of AChEl treatment and neurological or psychiatric disorders.

Patients with and without donepezil treatment were assessed using the following questionnaires: $\mathrm{K}$ MMSE to determine the severity of cognitive decline; $A D$ assessment scale-cognitive subscale (ADASCog) to establish the severity of cognitive and non-cognitive dysfunction from mild to severe AD; CDR to assess the severity of cognitive impairment; and geriatric depression scale (GDS) to evaluate the severity of depressed mood. The questionnaires were administered to patients with $\mathrm{MCl}$ before and after 6-month donepezil treatment. Mann-Whitney U-test was used to analyze the differences between healthy controls and patients with $\mathrm{MCl}$ as well as healthy controls and donepezil-treated patients. A Wilcoxon's signedrank test was used to compare the scores on K-MMSE, ADAS-Cog, CDR, and GDS before and after 6month donepezil treatment.

\section{Image acquisition}

All MRI data collected on 3T clinical scanner (Magnetom Tim Trio, Siemens Medical Solutions, Erlangen, Germany) using a head coil. Sagittal T1-weighted images were acquired using a 3-dimensional magnetization-prepared rapid acquisition gradient echo pulse sequence with the following parameters: repetition time $(T R)=1,700 \mathrm{~ms}$, echo time $(T E)=2.2 \mathrm{~ms}$, field of view $(F O V)=256 \times 256 \mathrm{~mm}^{2}$, matrix $=$ $512 \times 512$, slice thickness $=5 \mathrm{~mm}$, and slice gap $=2 \mathrm{~mm}$. Axial DTI were acquired using echo-planar imaging pulse sequence with the following parameters: TR: $5,200 \mathrm{~ms}, \mathrm{TE}=105 \mathrm{~ms}$, matrix $=128 \times 128$, and FOV $=220 \times 220 \mathrm{~mm}^{2}$. DTI consists of 24 directions $\left(b\right.$ factor $=1,000 \mathrm{~s} / \mathrm{mm}^{2}$ ) and 5 images without diffusion weighting ( $b$ factor $=0 \mathrm{~s} / \mathrm{mm}^{2}$ ). Phase-encoding was conducted in the anterior to posterior direction using a factor of 2 in-plane acceleration.

\section{Data processing and analysis}

T1-weighted images were analyzed with FreeSurfer v6.0 software (MGH, U.S.A., http://surfer.nmr.mgh.harvard.edu). DTI images were analyzed using Functional Magnetic Resonance Imaging of the Brain (FMRIB) Software Library (FSL) v6.0 software (Oxford, U.K; www.fmrib.ox.ac.uk/fsl). The Enhancing Neuroimaging Genetics through Meta-Analysis (ENIGMA) protocol was used to detect outliers and for visual inspection.

\section{Brain volume analysis}


Post-processing of T1 images entailed the following steps using the FreeSurfer segmentation pipeline ${ }^{6}$ : correction for head motion and non-uniformity of intensity, Talairach transformation of each subject's brain, removal of non-brain tissue, segmentation of cortical gray matter (GM), subcortical white matter (WM) and deep GM volumetric structures, triangular tessellation of the GM/WM interface and the GM/cerebrospinal fluid (CSF) boundary, and topology correction. Based on previous studies focused on $A D$, the brain regions of interest (ROIs) were selected as follows: superior/middle/inferior frontal gyrus (SFG/MFG/IFG), superior/middle/inferior temporal gyrus (STG/MTG/ITG), amygdala, caudate nucleus, hippocampus, putamen, and thalamus (Figure 1). These ROls were extracted for individual T1 imaging via automated parcellation of Freesurfer. Mann-Whitney Utest was used to compare brain volume between healthy controls vs. patients with $\mathrm{MCl}$, and a Wilcoxon signed-rank test was used to compare brain volume between patients treated with and without donepezil using SPSS (version 27.0, IBM, Armonk, NY, USA). The significance level was set to 0.05 after Bonferroni correction for the 11 ROIs to adjust for multiple comparisons (the level of significance after Bonferroni correction: $p<0.0046$ ).

\section{DTI scalars and WM connectivity analyses}

DTI pre-processing entailed skull removal and correction for motion and eddy currents ${ }^{16}$. Multiple DTI scalars (FA; fractional anisotropy, MD; mean diffusivity, RD; radial diffusivity, and AD; axial diffusivity) were generated for individual subject using the DTIFIT program that fits a DT model at each voxel of the diffusion images. The individual T1 images were rigidly registered to their corresponding non-diffusionweighted (B0) images using FMRIB's Linear Image Registration Tool (FLIRT) combined with mutual information cost function and trilinear interpolation ${ }^{16}$. The 11 ROls were extracted for each hemisphere in each subject's T1 imaging data via automated parcellation.

One patient showed motion artifact in the T1 images obtained after treatment, and thus $11 \mathrm{ROIs}$ in the patient were extracted in the T1 image obtained before treatment to register their T1 images with the diffusion space. We calculated the average values of FA, MD, RD, and AD in the 11 ROls of the 3 groups. To evaluate the structural connectivity, diffusion parameters were modeled using Bayesian Estimation of Diffusion Parameters Obtained using Sampling Techniques (BEDPOSTX) with crossing-fiber modeling ${ }^{16}$. The BEDPOSTX model of diffusion signal as ball (isotropic) and stick (anisotropic) components generates a distribution of likely fiber orientations within each voxel as well as an estimate of the uncertainty in these orientations ${ }^{20}$. We used FSL probabilistic tractography (connectivity modeling) to evaluate WM connectivity between seed (hippocampus) and target (10 ROIs) regions as follows: 5000 streamlines per each voxel in the thalamus, 0.2 curvature threshold, $0.5 \mathrm{~mm}$ step length, and loop check. The connectivity values were routinely thresholded at $10 \%$ to eliminate aberrant connections due to noise and error ${ }^{16,21}$. For the group analysis, a Mann-Whitney U-test was used to compare DTI scalars and WM connectivity between healthy controls and patients with $\mathrm{MCl}$. Wilcoxon's signed-rank test was used to compare DTI scalars and WM connectivity between patients treated with and without donepezil using SPSS (version 27.0, IBM, Armonk, NY, USA). The significance level was set to 0.05 after Bonferroni correction for the 10 to 11 ROls to adjust for multiple comparisons (the levels of significance after Bonferroni correction: $p<0.0046$ for DTI scalars and $p \leq 0.005$ for WM connectivity). 


\section{Results}

\section{Changes in symptom severity}

The average K-MMSE scores in healthy controls, untreated patients with $\mathrm{MCl}$ (baseline), and donepeziltreated patients (follow-up) were $28.6 \pm 1.1,16.5 \pm 4.9$, and $17.5 \pm 2.9$, respectively. The average K-MMSE score of patients with $\mathrm{MCl}$ was improved by $7.9 \%$ after 6 months of donepezil treatment $(\mathrm{p}=$ 0.031). Average ADAS-Cog scores in patients with $\mathrm{MCl}$ and treated patients were $25.6 \pm 6.2$ and $24.4 \pm$ 5.9 , respectively $(p=0.506)$; average CDR scores were $0.6 \pm 0.2$ and $0.6 \pm 0.2$, respectively $(p=0.317)$, and GDS scores were $13.2 \pm 5.2$ and $12.7 \pm 4.9$, respectively $(p=0.372)$.

\section{Brain volume changes}

Patients with $\mathrm{MCl}$ showed significantly decreased hippocampal volume compared with healthy controls $(p<0.05$, Bonferroni corrected) (Figures 2-3, Table 1). However, no significant differences were detected in the 11 ROls between patients with $\mathrm{MCl}$ and treated patients (Figure 3, Table 1).

\section{Changes in DTI scalars}

Compared with healthy controls, patients with $\mathrm{MCl}$ had higher MD $(p=0.003)$ and $\mathrm{RD}(\mathrm{p}=0.002)$ in the amygdala ( $p<0.05$, Bonferroni corrected) (Figure 4). Patients with $\mathrm{MCl}$ showed decreased FA in the hippocampus and amygdala ( $p \leq 0.05$, not corrected for multiple comparison) (Figure 2). None of the other ROls showed significant differences in MD and RD between healthy controls and patients with $\mathrm{MCl}$ (Supplemental Tables 1-4). In addition, no significant differences were found in the DTI scalars of the 11 ROls between patients with $\mathrm{MCl}$ and treated patients (Supplemental Tables 1-4).

\section{Hippocampal white matter connectivity}

Patients with $\mathrm{MCl}$ showed a significant decrease in hippocampal-SFG WM connectivity compared with healthy controls ( $p<0.05$, Bonferroni corrected) (Figure 2, Table 2). Following 6-month donepezil treatment, the patients with $\mathrm{MCl}$ showed increased hippocampal-ITG WM connectivity $(p<0.05$, Bonferroni corrected) (Figure 5, Table 2).

\section{Discussion}

\subsection{Summary of main findings}

Compared with healthy controls, patients with $\mathrm{MCl}$ showed decreased hippocampal volume and WM connectivity with the SFG, as well as increased MD and RD in the amygdala $(p<0.05$, Bonferronicorrected). Given that the hippocampal volume loss is consistent with evidence supporting AD diagnosis and tracking ${ }^{22,23}$. Further, patients with $\mathrm{MCl}$ showed enhanced MMSE scores and increased hippocampalITG connectivity ( $p<0.05$, Bonferroni-corrected) after 6-month donepezil treatment. These results suggest that increased hippocampal-ITG WM connectivity may be attributed to donepezil treatment. 


\subsection{Brain volume and DTI scalars in $\mathrm{MCl}$}

It is well known that hippocampus atrophy is at the core of AD pathophysiology. Patients with $\mathrm{MCl}$ showed a significant decrease in hippocampal volume compared with healthy controls. These results support the notion that hippocampal abnormalities are associated with early detection of $A D^{7,24-}$ 27. However, no volumetric increase across all the brain areas was detected after donepezil treatment.

Compared with healthy controls, patients with $\mathrm{MCl}$ showed higher $\mathrm{MD}$ and $\mathrm{RD}$ in the amygdala $(\mathrm{p}<0.05$, Bonferroni-corrected). Patients with $\mathrm{MCl}$ showed decreased FA in the hippocampus and amygdala $(\mathrm{p}<$ 0.05 , not corrected for multiple comparison), but the level of significance via multiple comparison correction was not high enough to validate this finding. A DTI study ${ }^{28}$ reported a decreased FA and a three-fold increase in trace value compared with MD in the hippocampus and amygdala of patients with $A D$ compared with healthy controls. A similar study ${ }^{29}$ also found a significantly elevated $M D$ in the hippocampus and amygdala of $A D$ patients. MD measures the average diffusivity in the non-colinear directions of free diffusion and RD quantifies the diffusion of water molecules in a direction perpendicular to the axon fibers ${ }^{30-32}$. The increased MD in the amygdala of patients with $\mathrm{MCl}$ was associated with an increase in free water diffusion and the increased RD was related to greater myelin damage. However, no change in DTI scalars in the all brain areas of patients was detected after donepezil treatment. Thus, alterations of hippocampal volume and DTI scalars in patients with $\mathrm{MCl}$ may be associated with early prediction of progression to $A D$.

\subsection{Structural connectivity in $\mathrm{MCl}$}

Structural connectivity is potentially important for the early diagnosis of AD. We found a decreased hippocampal-SFG WM connectivity in patients with $\mathrm{MCl}$ compared with healthy controls. This result, which has not been reported in previous structural connectivity studies, was consistent with that of a functional connectivity study ${ }^{33}$ suggesting that AD patients manifested decreased hippocampal-SFG connectivity compared with healthy controls. Another recent study ${ }^{34}$ showed decreased hippocampalSFG connectivity in MCl patients. The STG occupies the medial part of PFC (mPFC), which plays a critical role in multi-tasking, social cognition, attention, and emotion ${ }^{35}$. A 7T fMRI study ${ }^{36}$ revealed decreased hippocampal-SFG connectivity in AD, suggesting that lower MMSE scores were associated with reduced connectivity between the hippocampus and SFG. Thus, the decreased hippocampal-SFG WM connectivity is a potentially important biomarker for the early clinical diagnosis of $A D$.

\subsection{Structural connectivity after donepezil treatment in $\mathrm{MCl}$}

To our knowledge, this is the first study evaluating hippocampus-related structural connectivity in patients with $\mathrm{MCl}$ following donepezil treatment. In the current study, the MMSE scores of patients with $\mathrm{MCl}$ improved after donepezil treatment by $7.9 \%$. Additionally, patients with $\mathrm{MCl}$ showed increased hippocampal-ITG WM connectivity after 6 months of treatment. The ITG plays an important role in verbal fluency, a cognitive function affected early in the onset of $A D^{37}$. A study ${ }^{38}$ investigating the cognitive 
function of ITG in patients with $\mathrm{MCl}$ reported that MMSE scores are significant positively correlated with hippocampal-ITG connectivity. AD patients with a decline in the MMSE score following nine months of donepezil treatment showed decreased volume in the inferior temporal gyrus compared with increased $\mathrm{MMSE}^{39}$. Improved K-MMSE scores concomitant with increased hippocampal-ITG WM connectivity are potentially attributed to donepezil treatment.

Donepezil activates central cholinergic transmission and enhances the survival of newborn neurons in the hippocampal dentate gyrus ${ }^{40}$. Dong et al ${ }^{41}$ suggested that donepezil treatment reduced beta-amyloid plaques and increased synaptic density. Beta-amyloid deposition has been linked to AD pathology and induces multiple biochemical changes in cells including an increase in cytosolic calcium, which contributes to down-regulation of the expression of glutamate receptors in postsynaptic membrane ${ }^{42}$. Patients with early $A D$ showed an increase in serum concentration of brain-derived neurotrophic factor (BDNF) during donepezil treatment. BDNF belongs to the family of nerve growth factors and plays an important role in neuronal survival and synaptic plasticity in the central nervous system ${ }^{43}$. These findings provide evidence suggesting that hippocampal atrophy and decreased hippocampal-SFG WM connectivity may be closely related to AD pathogenesis and the increased hippocampal-ITG WM connectivity in donepezil-treated patients can be attributed to the treatment.

\subsection{Limitations and future directions}

This study has some limitations that should be mentioned. The small sample size does not ensure sufficiently high statistical power. To address this limitation, a statistical threshold of $P$ value less than 0.05 using Bonferroni correction was used. Another limitation is the short follow-up duration after donepezil treatment. Therefore, a placebo-controlled study of a large population of $\mathrm{MCl}$ patients and a long-term follow-up are needed to evaluate the time course of treatment change. In addition, such studies should investigate the changes in structural connectivity between mild and moderate $A D$ and between moderate and severe $A D$ in light of the effects of donepezil treatment.

\section{Conclusion}

This study demonstrates variations in WM connectivity after donepezil treatment in patients with $\mathrm{MCl}$. Increased K-MMSE scores and hippocampal-ITG WM connectivity in donepezil-treated patients can be attributed to treatment, suggesting that the hippocampal-ITG WM connectivity are a potentially important biomarker for donepezil treatment. These findings can be used to develop theories explaining the etiology of $\mathrm{MCl}$ and the mode of treatment using anticholinesterases.

\section{Declarations}

\section{Compliance with ethical standards}


This study approved by the Institutional Review Board (IRB) of Chonnam National University Hospital (CNUH). The experimental procedures and methods were performed in accordance with the relevant guidelines and regulations approved by IRB-CNUH. Informed consent form was obtained from each participant.

\section{Conflict of interest}

The authors declare that they have no conflicts of interest.

\section{Data Availability}

The data that support the findings of this study are available from the corresponding author, Gwang-Woo Jeong, upon reasonable request.

\section{Acknowledgments}

This research was supported by the grants from the National Research Foundation funded by the Korea government (MSIT; 2021R1C1C2011748, MSICT; 2018R1A2B2006260 and 2018R1C1B6005456) and the Chonnam National University (CNU) Research Fund for the CNU distinguished research professor (20172022).

Author contributions: G.W.K., K.S.P., and G.W.J. designed the study; G.W.K. and G.W.J performed the majority of experiments; G.W.K., K.S.P., and G.W.J contributed to the analysis and interpretation of results; G.W.K., K.S.P., and G.W.J wrote the first draft of the manuscript; G.W.J. has approved the final manuscript and completed manuscript; also, all authors agree with the content of the manuscript.

\section{References}

1. Morris, J. C. Mild cognitive impairment and preclinical Alzheimer's disease.Geriatrics Suppl,914(2005).

2. Gauthier, S. et al. Mild cognitive impairment., 367, 1262-1270 https://doi.org/10.1016/S01406736(06)68542-5 (2006).

3. Rankin, D. et al. Identifying Key Predictors of Cognitive Dysfunction in Older People Using Supervised Machine Learning Techniques: Observational Study. JMIR Med Inform, 8, e20995 https://doi.org/10.2196/20995 (2020).

4. Misra, C., Fan, Y. \& Davatzikos, C. Baseline and longitudinal patterns of brain atrophy in $\mathrm{MCl}$ patients, and their use in prediction of short-term conversion to AD: results from ADNI., 44, 1415-1422 https://doi.org/10.1016/j.neuroimage.2008.10.031 (2009).

5. Xue, J. et al. Altered Directed Functional Connectivity of the Hippocampus in Mild Cognitive Impairment and Alzheimer's Disease: A Resting-State fMRI Study. Front Aging Neurosci, 11, 326 https://doi.org/10.3389/fnagi.2019.00326 (2019). 
6. Kim, G. W., Kim, B. C., Park, K. S. \& Jeong, G. W. A pilot study of brain morphometry following donepezil treatment in mild cognitive impairment: volume changes of cortical/subcortical regions and hippocampal subfields. Sci Rep, 10, 10912 https://doi.org/10.1038/s41598-020-67873-y (2020).

7. Palesi, F. et al. DTI and MR Volumetry of Hippocampus-PC/PCC Circuit: In Search of Early Micro- and Macrostructural Signs of Alzheimers's Disease. Neurol Res Int 2012, 517876, doi:10.1155/2012/517876 (2012).

8. Beversdorf, D. Q., Nagaraja, H. N., Bornstein, R. A. \& Scharre, D. W. The Effect of Donepezil on Problem-solving Ability in Individuals With Amnestic Mild Cognitive Impairment: A Pilot Study. Cogn Behav Neurol, 34, 182-187 https://doi.org/10.1097/WNN.0000000000000280 (2021).

9. Cavedo, E. et al. Reduced basal forebrain atrophy progression in a randomized Donepezil trial in prodromal Alzheimer's disease. Sci Rep, 7, 11706 https://doi.org/10.1038/s41598-017-09780-3 (2017).

10. Kasa, P., Papp, H., Kasa, P. Jr. \& Torok, I. Donepezil dose-dependently inhibits acetylcholinesterase activity in various areas and in the presynaptic cholinergic and the postsynaptic cholinoceptive enzyme-positive structures in the human and rat brain., 101, 89-100 https://doi.org/10.1016/s03064522(00)00335-3 (2000).

11. Scali, C. et al. Effect of subchronic administration of metrifonate, rivastigmine and donepezil on brain acetylcholine in aged F344 rats. J Neural Transm (Vienna), 109, 1067-1080 https://doi.org/10.1007/s007020200090 (2002).

12. Risacher, S. L. et al. Cholinergic Enhancement of Brain Activation in Mild Cognitive Impairment during Episodic Memory Encoding. Front Psychiatry, 4, 105 https://doi.org/10.3389/fpsyt.2013.00105 (2013).

13. Petrella, J. R. et al. Effects of donepezil on cortical activation in mild cognitive impairment: a pilot double-blind placebo-controlled trial using functional MR imaging. AJNR Am J Neuroradiol, 30, 411416 https://doi.org/10.3174/ajnr.A1359 (2009).

14. La Rocca, M., Amoroso, N., Monaco, A., Bellotti, R. \& Tangaro, S. A novel approach to brain connectivity reveals early structural changes in Alzheimer's disease. Physiol Meas, 39, 074005 https://doi.org/10.1088/1361-6579/aacf1f (2018).

15. Caso, F. et al. White Matter Degeneration in Atypical Alzheimer Disease., 277, 162-172 https://doi.org/10.1148/radiol.2015142766 (2015).

16. Kim, G. W., Park, S. E., Park, K. \& Jeong, G. W. White Matter Connectivity and Gray Matter Volume Changes Following Donepezil Treatment in Patients With Mild Cognitive Impairment: A Preliminary Study Using Probabilistic Tractography. Front Aging Neurosci, 12, 604940 https://doi.org/10.3389/fnagi.2020.604940 (2021).

17. Jaimes, C. et al. Probabilistic tractography-based thalamic parcellation in healthy newborns and newborns with congenital heart disease. J Magn Reson Imaging, 47, 1626-1637 https://doi.org/10.1002/jmri.25875 (2018). 
18. King-Robson, J., Wilson, H. \& Politis, M. \& Alzheimer's Disease Neuroimaging, I. Associations Between Amyloid and Tau Pathology, and Connectome Alterations, in Alzheimer's Disease and Mild Cognitive Impairment. J Alzheimers Dis, 82, 541-560 https://doi.org/10.3233/JAD-201457 (2021).

19. Morris, J. C. Revised criteria for mild cognitive impairment may compromise the diagnosis of Alzheimer disease dementia. Arch Neurol, 69, 700-708 https://doi.org/10.1001/archneurol.2011.3152 (2012).

20. Theisen, F. et al. Evaluation of striatonigral connectivity using probabilistic tractography in Parkinson's disease. Neuroimage Clin, 16, 557-563 https://doi.org/10.1016/j.nicl.2017.09.009 (2017).

21. Cho, K. I. et al. Altered Thalamo-Cortical White Matter Connectivity: Probabilistic Tractography Study in Clinical-High Risk for Psychosis and First-Episode Psychosis. Schizophr Bull, 42, 723-731 https://doi.org/10.1093/schbul/sbv169 (2016).

22. Barnes, J. et al. A meta-analysis of hippocampal atrophy rates in Alzheimer's disease. Neurobiol Aging, 30, 1711-1723 https://doi.org/10.1016/j.neurobiolaging.2008.01.010 (2009).

23. Saribudak, A., Subick, A. A., Kim, N. H., Rutta, J. A. \& Uyar, M. U. Gene Expressions, Hippocampal Volume Loss, and MMSE Scores in Computation of Progression and Pharmacologic Therapy Effects for Alzheimer's Disease. IEEE/ACM Trans Comput Biol Bioinform, 17, 608-622 https://doi.org/10.1109/TCBB.2018.2870363 (2020).

24. Hashimoto, M. et al. Does donepezil treatment slow the progression of hippocampal atrophy in patients with Alzheimer's disease? Am J Psychiatry, 162, 676-682 https://doi.org/10.1176/appi.ajp.162.4.676 (2005).

25. He, J. et al. Differences in brain volume, hippocampal volume, cerebrovascular risk factors, and apolipoprotein E4 among mild cognitive impairment subtypes. Arch Neurol, 66, 1393-1399 https://doi.org/10.1001/archneurol.2009.252 (2009).

26. Laakso, M. P. et al. Volumes of hippocampus, amygdala and frontal lobes in the MRI-based diagnosis of early Alzheimer's disease: correlation with memory functions. J Neural Transm Park Dis Dement Sect, 9, 73-86 https://doi.org/10.1007/BF02252964 (1995).

27. Peng, G. P. et al. Correlation of hippocampal volume and cognitive performances in patients with either mild cognitive impairment or Alzheimer's disease. CNS Neurosci Ther, 21, 15-22 https://doi.org/10.1111/cns.12317 (2015).

28. Tang, X. et al. Shape and diffusion tensor imaging based integrative analysis of the hippocampus and the amygdala in Alzheimer's disease. Magn Reson Imaging, 34, 1087-1099 https://doi.org/10.1016/j.mri.2016.05.001 (2016).

29. Rose, S. E., Janke, A. L. \& Chalk, J. B. Gray and white matter changes in Alzheimer's disease: a diffusion tensor imaging study. J Magn Reson Imaging, 27, 20-26 https://doi.org/10.1002/jmri.21231 (2008).

30. Ceceli, A. O., Bradberry, C. W. \& Goldstein, R. Z. The neurobiology of drug addiction: cross-species insights into the dysfunction and recovery of the prefrontal cortex. Neuropsychopharmacology, 
https://doi.org/10.1038/s41386-021-01153-9 (2021).

31. Pierpaoli, C., Jezzard, P., Basser, P. J., Barnett, A. \& Di Chiro, G. Diffusion tensor MR imaging of the human brain., 201, 637-648 https://doi.org/10.1148/radiology.201.3.8939209 (1996).

32. Song, S. K. et al. Demyelination increases radial diffusivity in corpus callosum of mouse brain., 26 , 132-140 https://doi.org/10.1016/j.neuroimage.2005.01.028 (2005).

33. Wang, L. et al. Changes in hippocampal connectivity in the early stages of Alzheimer's disease: evidence from resting state fMRI., 31, 496-504 https://doi.org/10.1016/j.neuroimage.2005.12.033 (2006).

34. Berron, D., van Westen, D., Ossenkoppele, R., Strandberg, O. \& Hansson, O. Medial temporal lobe connectivity and its associations with cognition in early Alzheimer's disease., 143, 1233-1248 https://doi.org/10.1093/brain/awaa068 (2020).

35. Kraljevic, N. et al. Behavioral, Anatomical and Heritable Convergence of Affect and Cognition in Superior Frontal Cortex., 243, 118561 https://doi.org/10.1016/j.neuroimage.2021.118561 (2021).

36. Velayudhan, L. et al. Hippocampal functional connectivity in Alzheimer's disease: a resting state 7T fMRI study. Int Psychogeriatr, 33, 95-96 https://doi.org/10.1017/S1041610220003440 (2021).

37. Scheff, S. W., Price, D. A., Schmitt, F. A., Scheff, M. A. \& Mufson, E. J. Synaptic loss in the inferior temporal gyrus in mild cognitive impairment and Alzheimer's disease. J Alzheimers Dis, 24, 547-557 https://doi.org/10.3233/JAD-2011-101782 (2011).

38. Wang, Z. et al. Baseline and longitudinal patterns of hippocampal connectivity in mild cognitive impairment: evidence from resting state fMRI. J Neurol Sci, 309, 79-85 https://doi.org/10.1016/j.jns.2011.07.017 (2011).

39. Bottini, G. et al. GOOD or BAD responder? Behavioural and neuroanatomical markers of clinical response to donepezil in dementia. Behav Neurol, 25, 61-72 (2012).

40. Kotani, S., Yamauchi, T., Teramoto, T. \& Ogura, H. Donepezil, an acetylcholinesterase inhibitor, enhances adult hippocampal neurogenesis. Chem Biol Interact, 175, 227-230 https://doi.org/10.1016/j.cbi.2008.04.004 (2008).

41. Dong, H., Yuede, C. M., Coughlan, C. A., Murphy, K. M. \& Csernansky, J. G. Effects of donepezil on amyloid-beta and synapse density in the Tg2576 mouse model of Alzheimer's disease. Brain Res, 1303, 169-178 https://doi.org/10.1016/j.brainres.2009.09.097 (2009).

42. Liu, S. J., Gasperini, R., Foa, L. \& Small, D. H. Amyloid-beta decreases cell-surface AMPA receptors by increasing intracellular calcium and phosphorylation of GluR2. J Alzheimers Dis, 21, 655-666 https://doi.org/10.3233/JAD-2010-091654 (2010).

43. Leyhe, T., Stransky, E., Eschweiler, G. W., Buchkremer, G. \& Laske, C. Increase of BDNF serum concentration during donepezil treatment of patients with early Alzheimer's disease. Eur Arch Psychiatry Clin Neurosci, 258, 124-128 https://doi.org/10.1007/s00406-007-0764-9 (2008).

\section{Tables}


Due to technical limitations,Tables 1 and 2 are only available as a download in the Supplemental Files section.

\section{Figures}

a

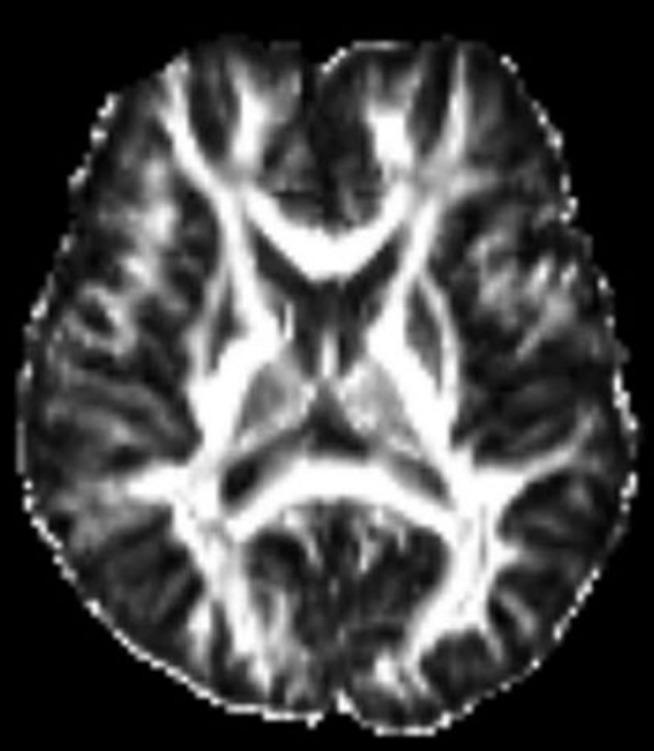

C

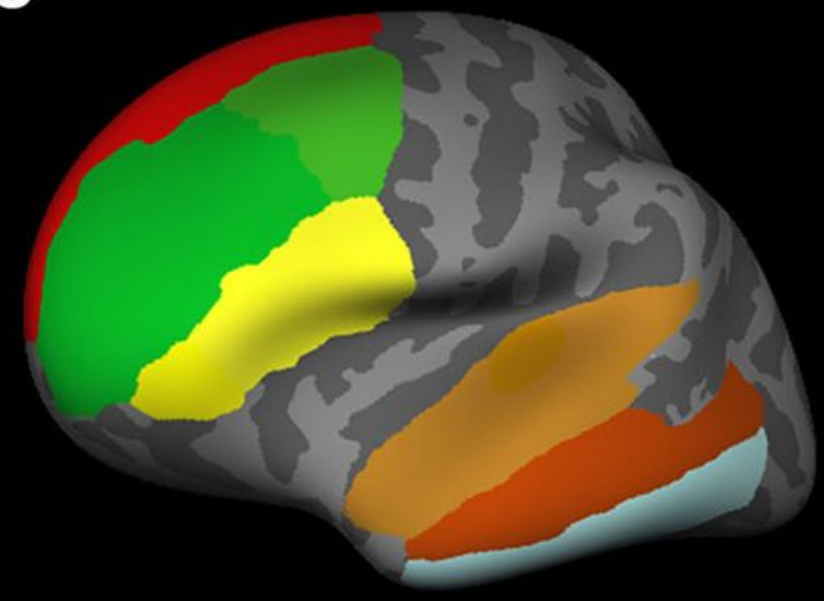

b

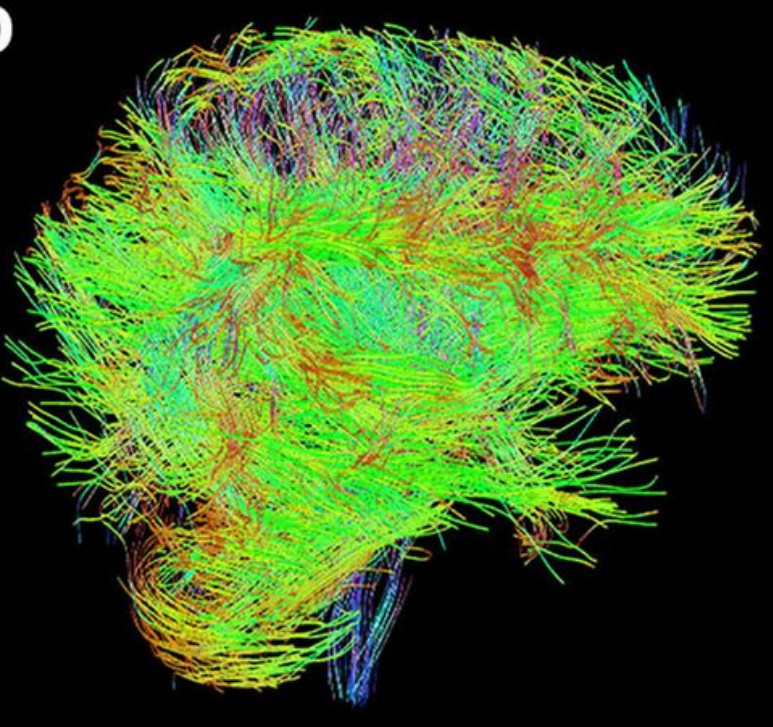

d
SFG
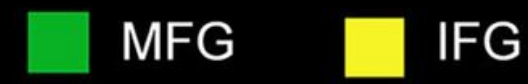

IFG
MTG

$\square$ ITG

\section{Hippocampus \\ Putamen \\ Amygdala \\ $\mathrm{CN}$}

\section{Hippocampus}

STG

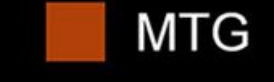


https://surfer.nmr.mgh.harvard.edu) and Microsoft Powerpoint (version 16 https://www.microsoft.com). SFG; superior frontal gyrus, MFG; middle frontal gyrus, IFG; inferior frontal gyrus, STG; superior temporal gyrus, MTG; middle temporal gyrus, ITG; inferior temporal gyrus, CN; caudate nucleus.

a

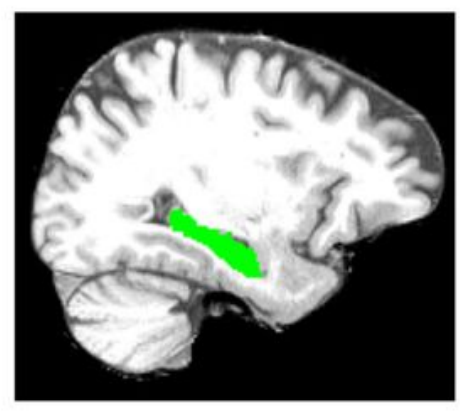

T1 image

b

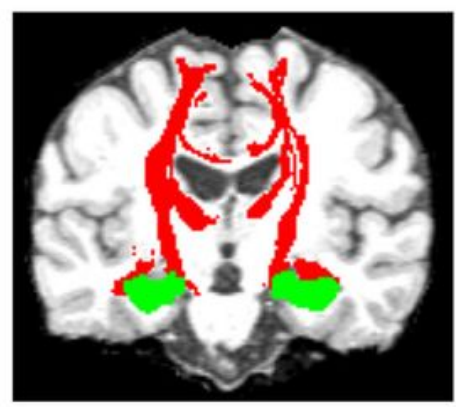

WM connectivity

C

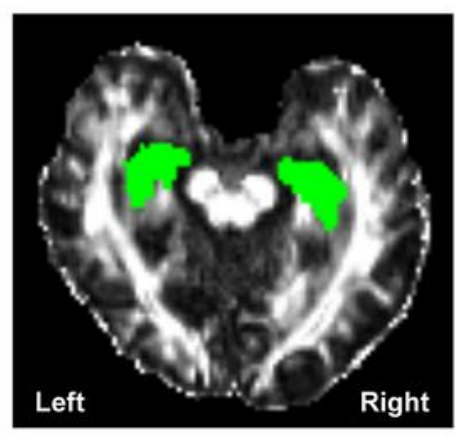

FA
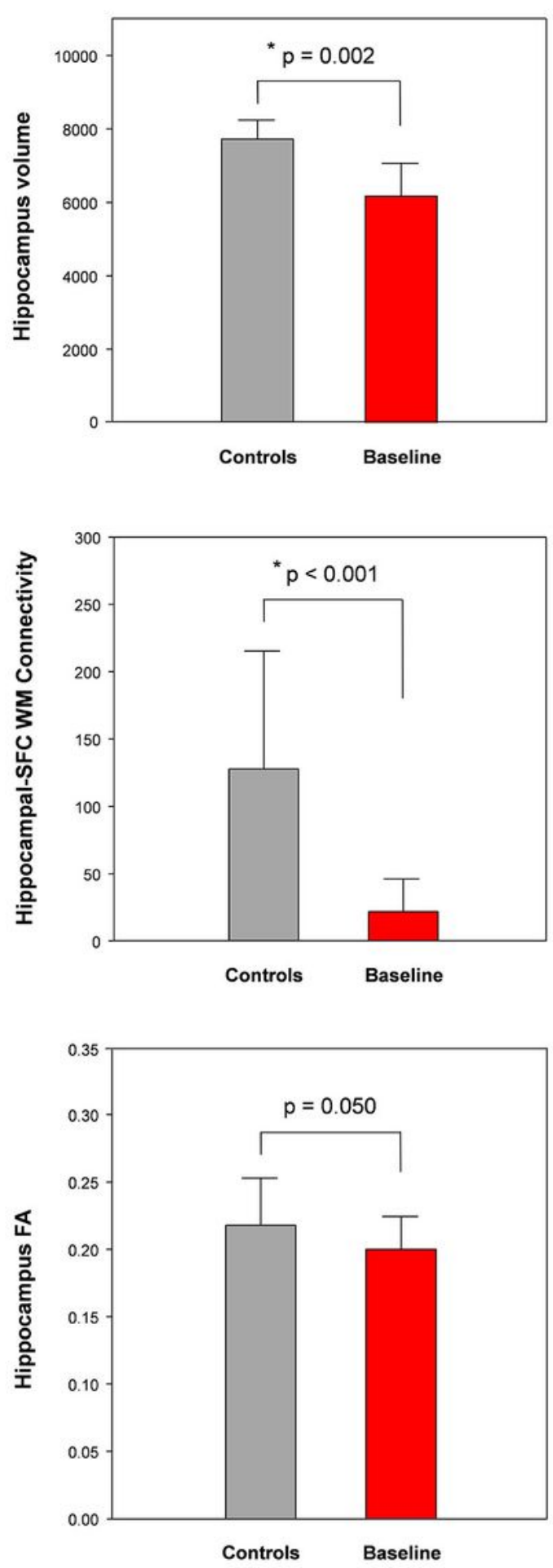

Figure 2

The decreased hippocampal volume (a) and hippocampal-superior frontal cortex (SFG) white matter (WM) connectivity (b) in the patients with $\mathrm{MCl}$ (baseline) compared with healthy controls $(p<0.05$, 
Bonferroni-corrected). Patients with $\mathrm{MCl}$ showed decreased fractional anisotropy (FA) in the hippocampus ( $p=0.05$, not corrected for multiple comparison) (c). Green in the left figure; hippocampal seed ROI, Red in left figure; WM connectivity. This figure was created using Freesurfer (version 6.0 https://surfer.nmr.mgh.harvard.edu), MRIcron (version 6 https://www.nitrc.org/projects/mricron), and Microsoft Powerpoint (version 16 https://www.microsoft.com). * significant difference (Bonferroni corrected, $\mathrm{p}<0.05)$.

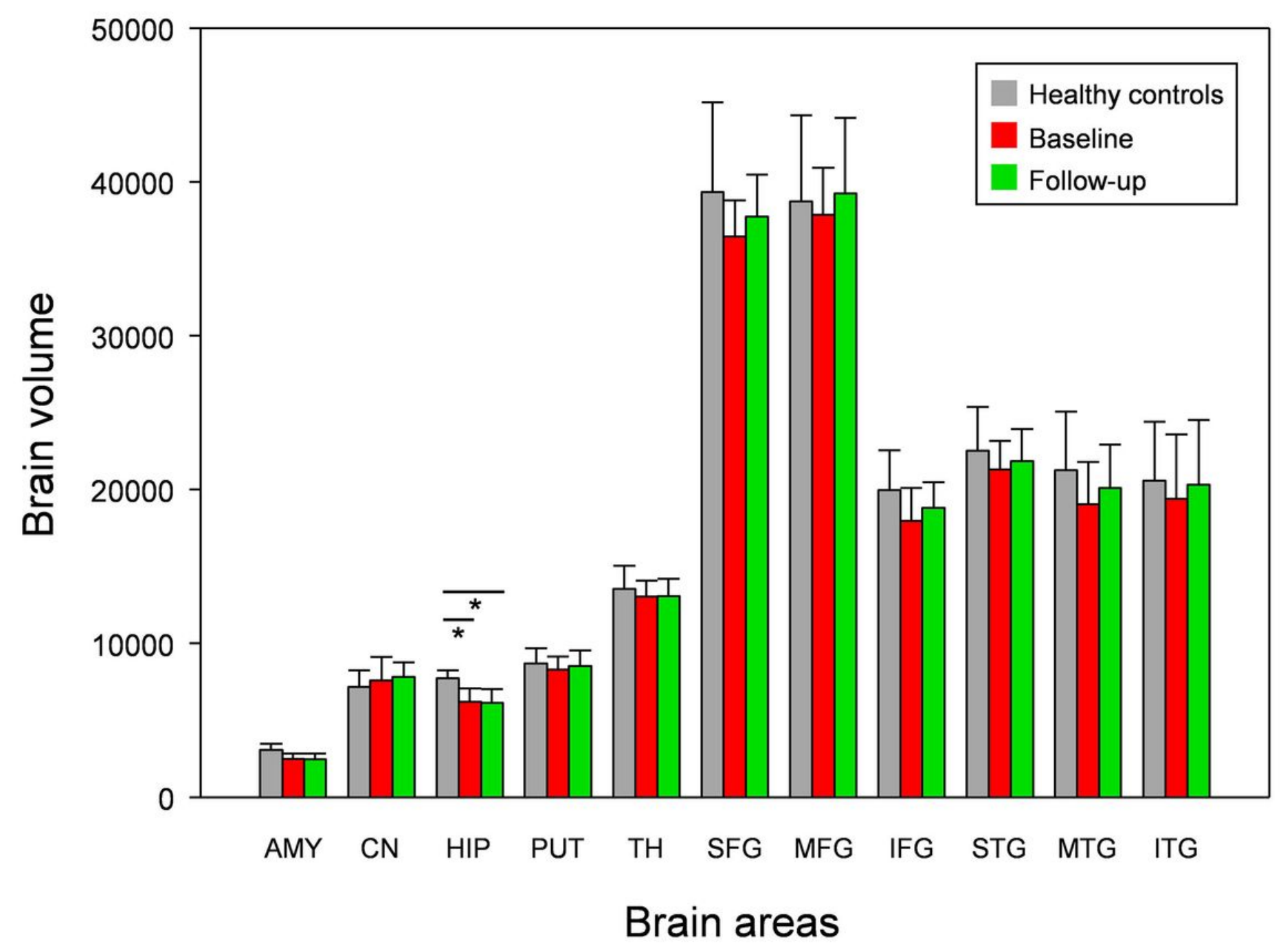

Figure 3

Mean brain volume in the 11 ROls in patients with $\mathrm{MCl}$ (baseline), donepezil-treated patients (follow-up), and healthy controls. However, no significant differences were detected in the 11 ROIs between patients with $\mathrm{MCl}$ and treated patients. AMY; amygdala, CN; caudate nucleus, HIP; hippocampus, PUT; putamen, SFG; superior frontal gyrus, MFG; middle frontal gyrus, IFG; inferior frontal gyrus, STG; superior temporal gyrus, MTG; middle temporal gyrus, ITG; inferior temporal gyrus. This figure was created using SigmaPlot (version 13 https://systatsoftware.com/products/sigmaplot) and Microsoft Powerpoint (version 16 https://www.microsoft.com). * significant difference (Bonferroni corrected, $p<0.05$ ). 
a

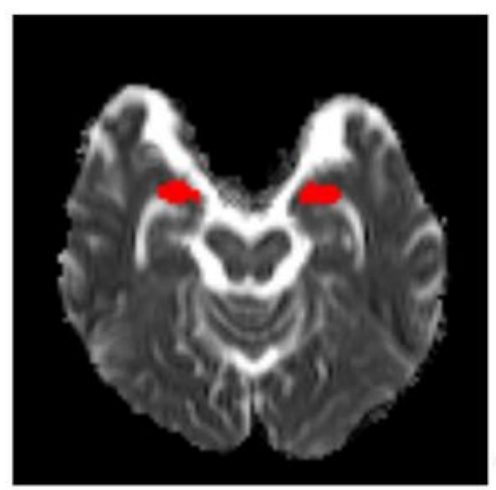

MD

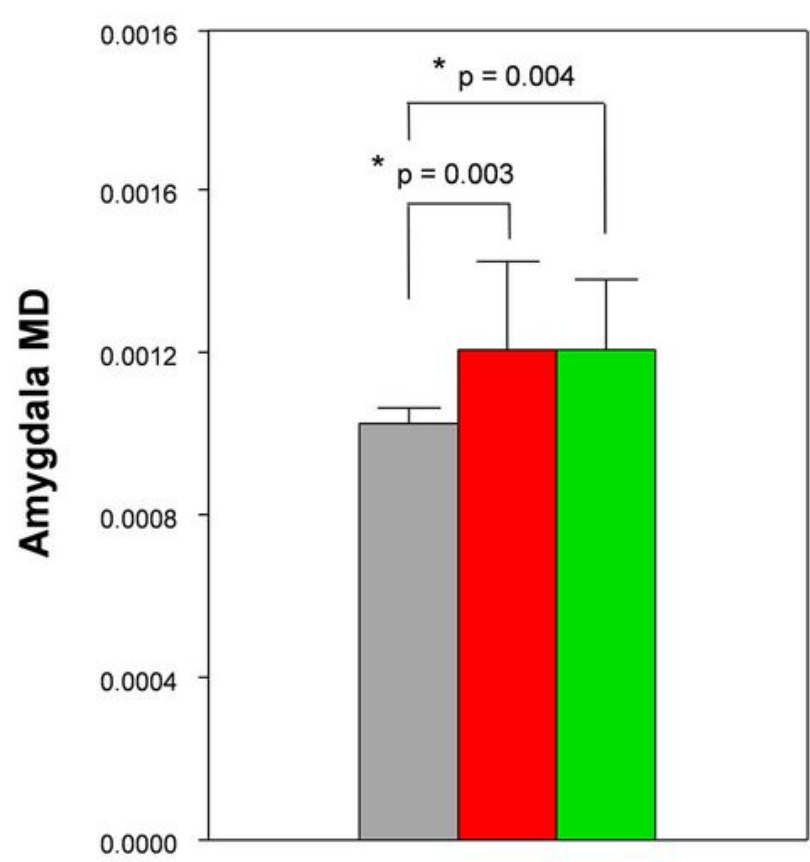

b

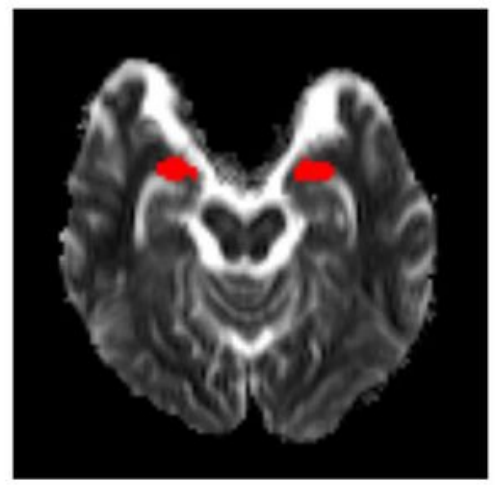

RD

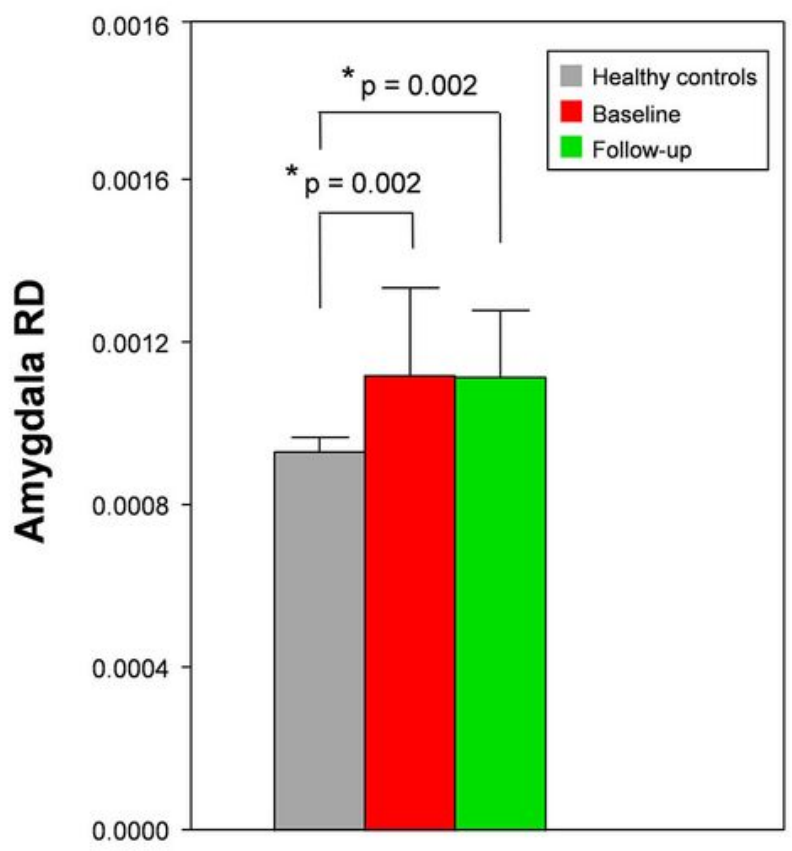

\section{Figure 4}

Increased mean diffusivity (MD) (a) and radial diffusivity (RD) (b) in the amygdala in the patients with $\mathrm{MCl}$ compared with healthy controls ( $\mathrm{p}<0.05$, Bonferroni corrected). None of the other $10 \mathrm{ROIs}$ showed significant differences in the DTI scalars between healthy controls and patients with $\mathrm{MCl}$. In addition, no significant differences were found in the $11 \mathrm{RO}$ s between patients with $\mathrm{MCl}$ and treated patients. Red in the left figure; the amygdala ROI. This figure was created using SigmaPlot (version 13 
https://systatsoftware.com/products/sigmaplot) and Microsoft Powerpoint (version 16 https://www.microsoft.com). * significant difference (Bonferroni corrected, $p<0.05$ ).

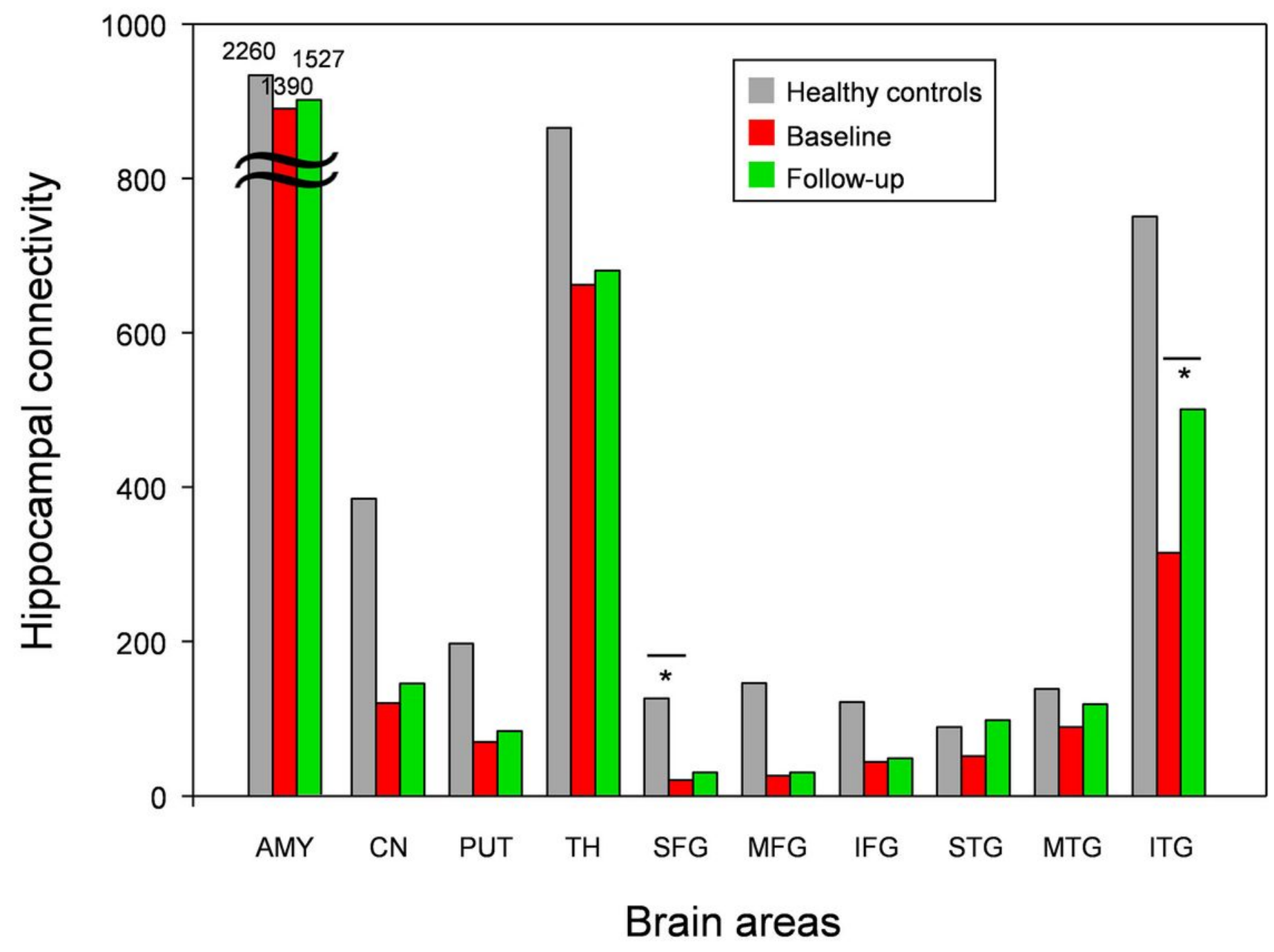

Figure 5

Mean white matter connectivity (WM) between the hippocampus (seed region) and the 10 ROIs in patients with $\mathrm{MCl}$ (baseline), donepezil-treated patients (follow-up), and healthy controls. Patients with $\mathrm{MCl}$ showed a significant decrease in the hippocampal-SFG WM connectivity compared with healthy controls ( $p<0.05$, Bonferroni corrected). Following 6-month donepezil treatment, the patients with $\mathrm{MCl}$ showed increased hippocampal-ITG WM connectivity ( $p<0.05$, Bonferroni corrected). AMY; amygdala, CN; caudate nucleus, HIP; hippocampus, PUT; putamen, SFG; superior frontal gyrus, MFG; middle frontal gyrus, IFG; inferior frontal gyrus, STG; superior temporal gyrus, MTG; middle temporal gyrus, ITG; inferior temporal gyrus. This figure was created using SigmaPlot (version 13 https://systatsoftware.com/products/sigmaplot) and Microsoft Powerpoint (version 16 https://www.microsoft.com). * significant difference (Bonferroni corrected, $p<0.05$ ).

\section{Supplementary Files}


This is a list of supplementary files associated with this preprint. Click to download.

- Tables.docx

- SupplementalMaterial.docx 